\title{
A new method to generate dust with astrophysical properties
}

J. F. Hansen, W. van Breugel, E. M. Bringa, G. A. Graham, B. A. Remington, E. A. Taylor, A. G. Tielens

April 30, 2010

8th International Conference on High Energy Density Laboratory Astrophysics Pasadena, CA, United States March 15, 2010 through March 18, 2010 
This document was prepared as an account of work sponsored by an agency of the United States government. Neither the United States government nor Lawrence Livermore National Security, LLC, nor any of their employees makes any warranty, expressed or implied, or assumes any legal liability or responsibility for the accuracy, completeness, or usefulness of any information, apparatus, product, or process disclosed, or represents that its use would not infringe privately owned rights. Reference herein to any specific commercial product, process, or service by trade name, trademark, manufacturer, or otherwise does not necessarily constitute or imply its endorsement, recommendation, or favoring by the United States government or Lawrence Livermore National Security, LLC. The views and opinions of authors expressed herein do not necessarily state or reflect those of the United States government or Lawrence Livermore National Security, LLC, and shall not be used for advertising or product endorsement purposes. 
Not to appear in Nonlearned J., 45.

\title{
A new method to generate dust with astrophysical properties
}

\author{
J. F. Hansen ${ }^{1}$ and Wil van Breugel ${ }^{2}$ and E. M. Bringa ${ }^{3}$ and G. A. Graham ${ }^{4}$ and B. A. \\ Remington \\ Lawrence Livermore National Laboratory, Livermore, CA \\ E. A. Taylor \\ Open University, Milton Keynes, United Kingdom ${ }^{5}$ \\ and \\ A. G. G. M. Tielens ${ }^{6}$ \\ NASA Ames Research Center, Mountain View, CA
}

\begin{abstract}
In interstellar and interplanetary space, the size distribution and composition of dust grains play an important role. For example, dust grains determine optical and ultraviolet extinction levels in astronomical observations, dominate the cooling rate of our Galaxy, and sets the thermal balance and radiative cooling rates in molecular clouds, which are the birth place of stars. Dust grains are also a source of damage and failure to space hardware and thus present a hazard to space flight. To model the size distribution and composition of dust grains, and their effect in the above scenarios, it is vital to understand the mechanism of dust-shock interaction. We demonstrate a new experiment which employs a laser
\end{abstract}

\footnotetext{
${ }^{1}$ current affiliation: General Atomics, 3550 General Atomics Court, San Diego, CA 92121; telephone: +1-925-424-6977; email: hansen46@llnl.gov

${ }^{2}$ current affiliation: University of California Merced, Merced, CA.

${ }^{3}$ current affiliation: Universidad Nacional de Cuyo, Mendoza, Argentina

${ }^{4}$ current affiliation: Natural History Museum, London, United Kingdom

${ }^{5}$ current affiliation:

${ }^{6}$ current affiliation: Leiden Observatory, Leiden, The Netherlands
} 
to subject dust grains to pressure spikes similar to those of colliding astrophysical dust, and which accelerates the grains to astrophysical velocities. The new method generates much larger data sets than earlier methods; we show how large quantities (thousands) of grains are accelerated at once, rather than accelerating individual grains, as is the case of earlier methods using electric fields.

Subject headings: dust grains: general — dust grains: shock processing — dust grains: size distribution — dust grains: composition — dust grains: cratering space hardware: damage — laboratory astrophysics

\section{Background: dust grains in astrophysics}

In this manuscript we present a new technique that can be employed to accelerate dust grains to hyper-velocities, and which subjects the dust grains to the short pressure spikes seen in grain-grain collisions in interstellar space. This new technique can become a useful tool for research on both interstellar dust grains and on hyper-velocity impacts of dust grains on space hardware.

The dust grain size distribution in interstellar space (1) is important because it determines the (a) optical and ultraviolet extinction levels in astronomical observations, thus affecting most observations from which conclusions about the Universe are drawn, (b) the observed depletions in interstellar space of "higher-Z" elements (C, Mg, Fe, Si, O, etc.), and (c) the thermal balance and radiative cooling rates in molecular clouds, which are the birthing place of stars $(2 ; 3 ; 4 ; 5 ; 6 ; 7 ; 8)$ and also dominates the cooling rate of our Galaxy. Furthermore, dust grains play an important role in the formation of most astrophysical objects from black holes down to meteorites (including stars and planets), and are also of great interest in astrochemistry and astrobiology.

Clearly then, understanding the size distribution and composition of astrophysical dust grains is important. The dominant mechanism determining the distribution is the shock processing occuring during grain-grain collisions $(6 ; 8)$ at very high relative velocities, illustrated schematically in Fig. 1. Grain composition can also be altered through phase transitions. As an example, one intriguing aspect is the "diamonds in the sky" prediction, whereby graphite grains are suggested to be transformed into diamonds in the strong shocks induced in grain-grain collisions (4). Adding support to this hypothesis was the discovery

of small diamond grains $(\sim 50 \AA)$ in carbonaceous meteorites, with a suggested interstellar origin (9). The shock strengths required are strong, $p_{\text {shock }}>500 \mathrm{kbar}$, but their durations are fleetingly short, a few picoseconds, and the spatial scales of the grains are sub-micron. 


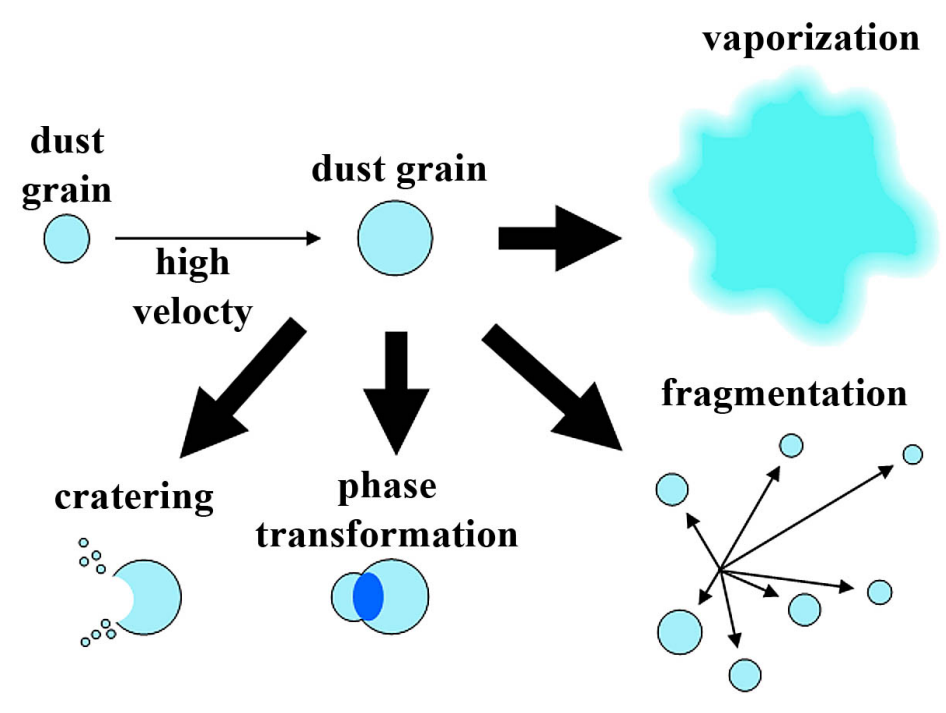

Fig. 1.- A grain-grain collision can result in (depending on the kinetic energy, material properties, and grain sizes involved) cratering, phase transformation, fragmentation, or vaporization.

Under these conditions, it is not known whether carbon can be transformed to diamond, as suggested. This prediction can be tested experimentally, provided the required pressure, spatial, and temporal scales can be reproduced.

Closer to home, micrometeorites and hypervelocity $(>1 \mathrm{~km} / \mathrm{s})$ interplanetary dust particles (IDPs) present a hazard to space flight and are a source of damage and failure to space hardware. Space-based hardware (solar panels, thermal shields, etc.) needs to be designed to operate for extended exposures in space, which means exposures to the presence of hypervelocity impacts of IDPs. Solar panels and other hardware from, for example, the Hubble Space Telescope (HST), that have been returned to Earth, exhibit very clearly the effects (cratering, micro-cracking, and other localized damage spots) of these IDP particle impacts $(10 ; 11 ; 12 ; 13 ; 14 ; 15 ; 16 ; 17)$. An example is shown in Fig. 2. An enduring puzzle, however, is why no impact craters smaller than $\sim 10 \mu \mathrm{m}$ are observed. The predicted IDP grain size distribution (1) suggests that the incident flux should in fact be largest for the smallest grains, and crater size should scale as some power of incident particle kinetic energy. Yet, there is little evidence of lower-kinetic energy impacts. Either the models for the size distribution of the incoming flux of IDPs are in error, or the effect of their impacts is not properly understood. This question can also be addressed experimentally, where the impactor mass and velocity can be controlled independently and varied over a considerable range. 


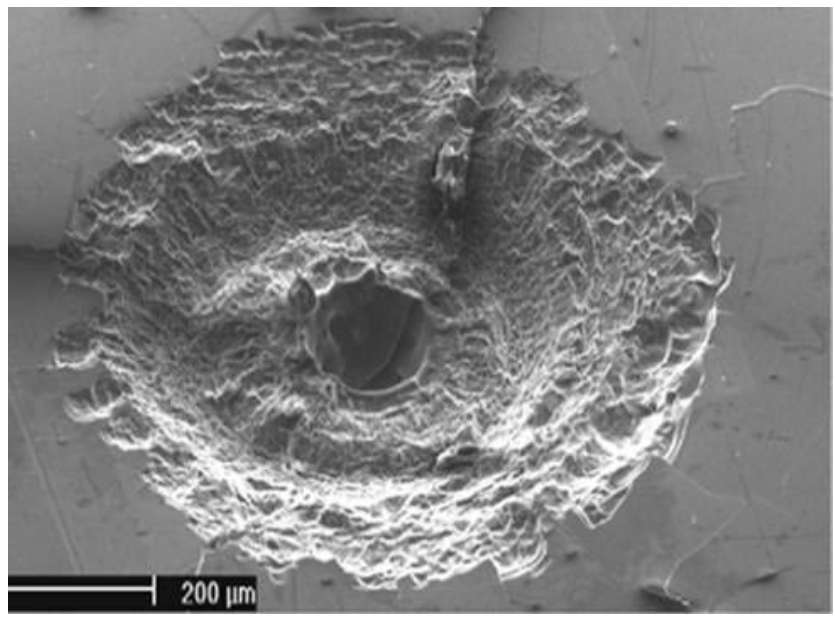

Fig. 2.- A scanning electron microscope (SEM) image of a crater on one of the solar panels of the Hubble Space Telescope. [Image courtesy Giles Graham.]

\section{Experiment design}

We have invented and tested a new laser-based laboratory capability to carry out experiments on the shock processing resulting from grain-grain collisions, and the damage and cratering of space hardware due to hypervelocity IDP impacts. To this end, we designed a laser target containing a collection of dust grains, as shown in Fig. 3. A laser illuminates a solid-density ablator $\sim 20 \mu \mathrm{m}$ thick, launching a strong shock which moves through the $0.1 \mathrm{~g} / \mathrm{cm}^{3}, \sim 200 \mu \mathrm{m}$ thick foam reservoir. At the back side of this reservoir are the dust particles of interest, many microns to sub-micron-size spheres of graphite, diamond, or other materials of interest to interstellar dust dynamics. As the shock releases at the back side of the reservoir the duration of high pressure conditions on the grains would be very short.

To guide the design, we show in Fig. 4 the results from numerical simulations in 1D using the HyADES radiation hydrodynamics code (18). (In this approximation, the grains are treated as a 1D very thin foil.) It is clear that we can control both the magnitude of the pressure spike from the shock and its duration in the dust grains. Figure 4 (top) shows pressure versus time inside a graphite grain. The predicted dwell time (high pressure interval) is $50 \mathrm{ps}$ or less. This is an over-estimate, due to the effect of artificial viscosity in the hydrodynamics code (the numerical "trick" to artificially spread out the shock discontinuity over several zones). A more time consuming MD simulation would give a considerably shorter, more realistic dwell time; for comparison see Fig. 5 which shows MD simulations albeit for smaller dust grains. 


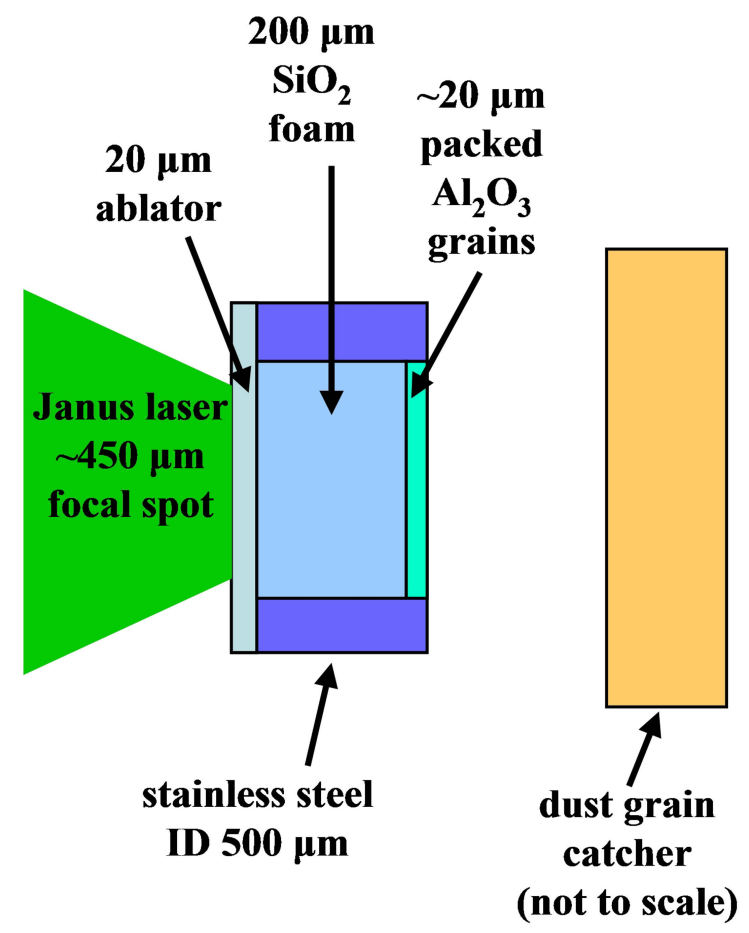

Fig. 3.- Target design: a $220 \mu \mathrm{m}$ thick stainless steel target substrate with a $500 \mu \mathrm{m}$ diameter hole is filled with aerogel. Coated on "top" of the target substrate (to the left in this image) is a $\sim 20 \mu \mathrm{m}$ thick ablator. The "bottom" of the hole (to the right in this image) contains a layer of $\mathrm{Al}_{2} \mathrm{O}_{3}$ grains. The layer thickness was nominally $20 \mu \mathrm{m}$ and the diameter of individual dust grains was $5 \mu \mathrm{m}$. 

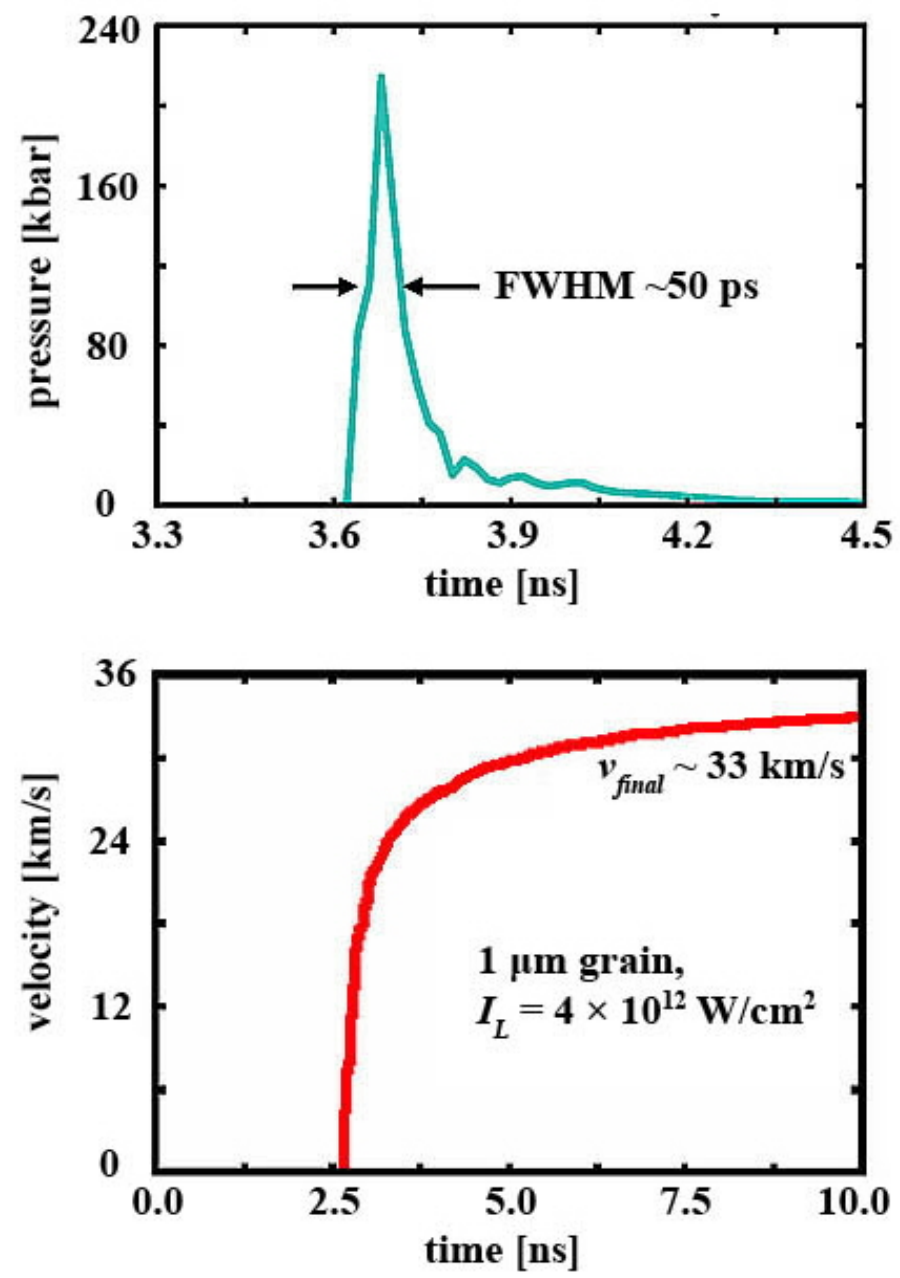

Fig. 4.- Pressure (top) and velocity (bottom) histories of a dust grain at the rear surface of the laser target as calculated in 1D HYADES simulations. 


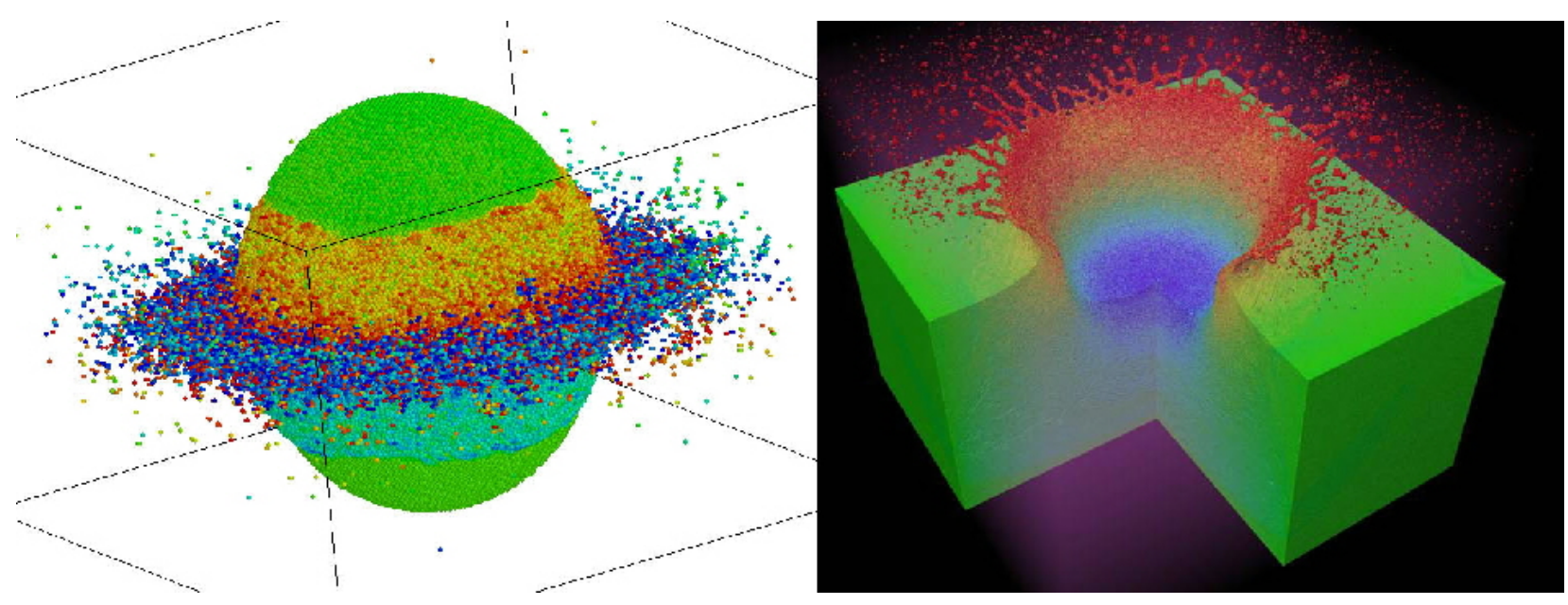

Fig. 5.- Two molecular dynamics (MD) simulations: Left: a grain-grain collision of the type that might be found in the flux of interplanetary dust. The relative impact velocity was $7 \mathrm{~km} / \mathrm{s}$ and the dust particles were $18 \mathrm{~nm}$ diameter $\mathrm{Cu}$ spheres. The resulting pressure spike felt by the $\mathrm{Cu}$ "dust grain" is $\sim 200 \mathrm{kbar}$ in magnitude with a duration $t_{F W H M} \approx 5$ ps. Right: a $20 \mathrm{~nm}$ grain $20 \mathrm{ps}$ after impacting a slab of material creating a crater. A color version of this image is available on-line. In both images, color represents potential energy $(\mathrm{PE})$ : bright red and dark blue mean high $\mathrm{PE}$, that is, large disorder. Green represents the PE minimum of the initial crystalline state. These MD simulations are state-of-the-art, yet very uncertain. They can be tested with the experiments that we have begun. [MD simulation courtesy Eduardo Bringa.]

Figure 4 (bottom) shows a predicted micro-impactor velocity of $\sim 30 \mathrm{~km} / \mathrm{s}$, the highest velocity we would expect based on our 1D HyADES simulation. Note that this velocity likely is an over-estimate by the $1 \mathrm{D}$ code, and corresponds better to the final velocity of the exfoam plasma than the final velocity of a dust grain; in a real 3D environment some plasma will slip past the grain instead of pushing it, and the final grain velocity will be reduced by a factor of a few.

\section{Experimental results}

To test our idea, we assembled and shot nine targets. Each target consisted of a piece of $220 \mu \mathrm{m}$ thick stainless steel with a drilled $500 \mu \mathrm{m}$ diameter hole filled with aerogel, as shown in Fig. 3. The "bottom" of the hole contained a layer of $5 \mu \mathrm{m}$ diameter $\mathrm{Al}_{2} \mathrm{O}_{3}$ grains. The layer thickness was nominally $20 \mu \mathrm{m}$ thick, although there were individual variations 
between each target (see Fig. 6). Coated on top of the shim stock was an ablator consisting of a $25.5 \mu \mathrm{m}$ thick layer of parylene-C, followed by a flash coating of $\sim 1000 \AA \mathrm{Al}$.

The experiment took place in the vacuum chamber of Target Room 1 at the Jupiter Laser Facility (19), using one beam of the Janus laser to drive the target. The laser wavelength was $527 \mathrm{~nm}$ and the laser pulse length was $5 \mathrm{~ns}$. The laser energy varied from shot to shot but was typically $200 \mathrm{~J}$. The laser focal spot diameter was $\sim 450 \mu \mathrm{m}$. A $25 \mathrm{~mm}$ by $50 \mathrm{~mm} \mathrm{Cu}$ foil was placed $25 \mathrm{~mm}$ behind the target to catch hyper-velocity grains. The foil was $125 \mu \mathrm{m}$ thick, $99.9 \%$ pure, "half hard" $\mathrm{Cu}$, and was backed by a thicker piece of $\mathrm{Cu}$.

Each of the nine targets produced craters indicative of hyper-velocity impacts on the $\mathrm{Cu}$ grain catcher foils. Viewing the foils with a scanning electron microscope (SEM) reveals a spectacular amount of craters. This is readily seen in the low magnification of Fig. 7. This suggests that we could have used fewer dust grains. Fortunately, most craters appear to be single impacts, caused by a single impactor. Some the craters are "classic" craters and some are shallower "splash" craters. Many craters contain traces of $\mathrm{Al}$ and $\mathrm{O}$, a strong indication that these craters were formed from grain impacts (see Fig. 8). These craters are not much bigger than $5 \mu \mathrm{m}$ in diameter, which also suggest they came from dust grain impacts. In some areas of the $\mathrm{Cu}$ foils, other craters show traces of Fe (see Fig. 9), likely due to impacts from steel droplets (from the target hole walls).

\section{Conclusions}

Using a new laser-based laboratory capability, we have subjected dust grains to pressure spikes similar to those of astro-physical dust processing, and have accelerated the dust grains to hyper-velocities. The dust grain composition in our experiment was $\mathrm{Al}_{2} \mathrm{O}_{3}$, but the new technique presented here should be tunable to diamond grains, "aggregate grains," and other grains of astrophysical interest, to study phase transitions and changes in grain size distribution. We have showed that the hyper-velocity grains caused cratering on a $\mathrm{Cu}$ foil in our experiment. Replacing the $\mathrm{Cu}$ foil with actual space hardware is easily accomplished, and a range of projectile grain sizes and velocities could be used, allowing the hardware surfaces to be examined for cratering and thermally driven micro-cracking. The dust grains can also be captured gently in aerogels, reproducing the methodology of the recent Stardust mission (20) and building on the experience of extracting and characterizing micrograin materials impacted into $\mathrm{SiO}_{2}$ aerogel $(13 ; 17)$. Observables to look for in future experiments include whether these shock processed micrograins (1) melted and refroze, or remained solid throughout, (2) fractured or remained whole, (3) suffered severe plastic flow, or remained largely elastic, i.e., retained their original shape, and (4) whether graphite micrograins transitioned 

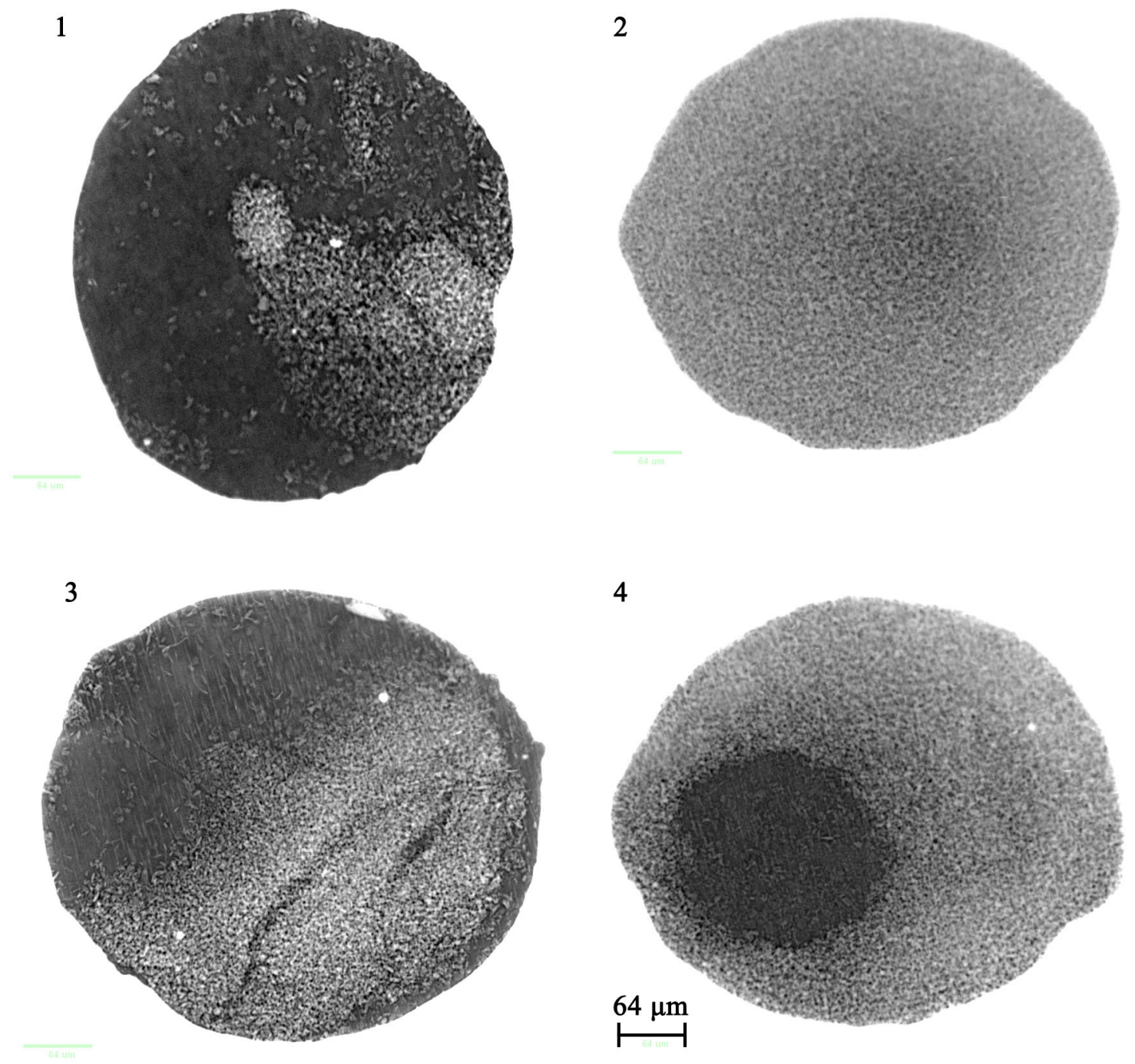

Fig. 6. - X-ray metrology images of four targets looking through the $500 \mu \mathrm{m}$ hole in the stainless steel substrate. The grain coverage over the hole varies from target to target; bright areas contain $\mathrm{Al}_{2} \mathrm{O}_{3}$ grains, while dark areas are free of grains. 


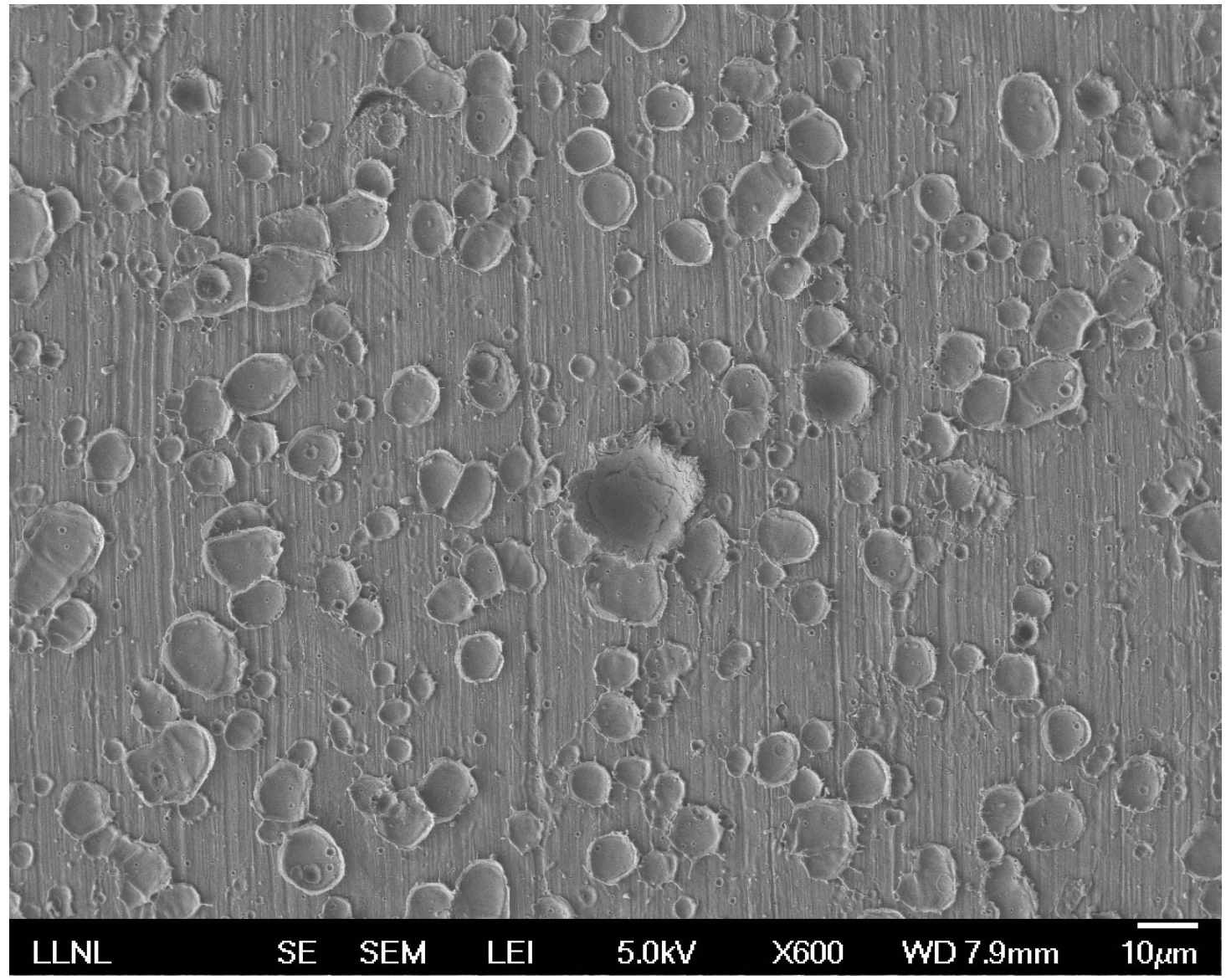

Fig. 7.- Image of a cratered $\mathrm{Cu}$ foil grain catcher showing an abundance of craters. [The image is a lower secondary electron image (LEI) produced by a scanning electron microscope (SEM) at $600 \times$ magnification.] 


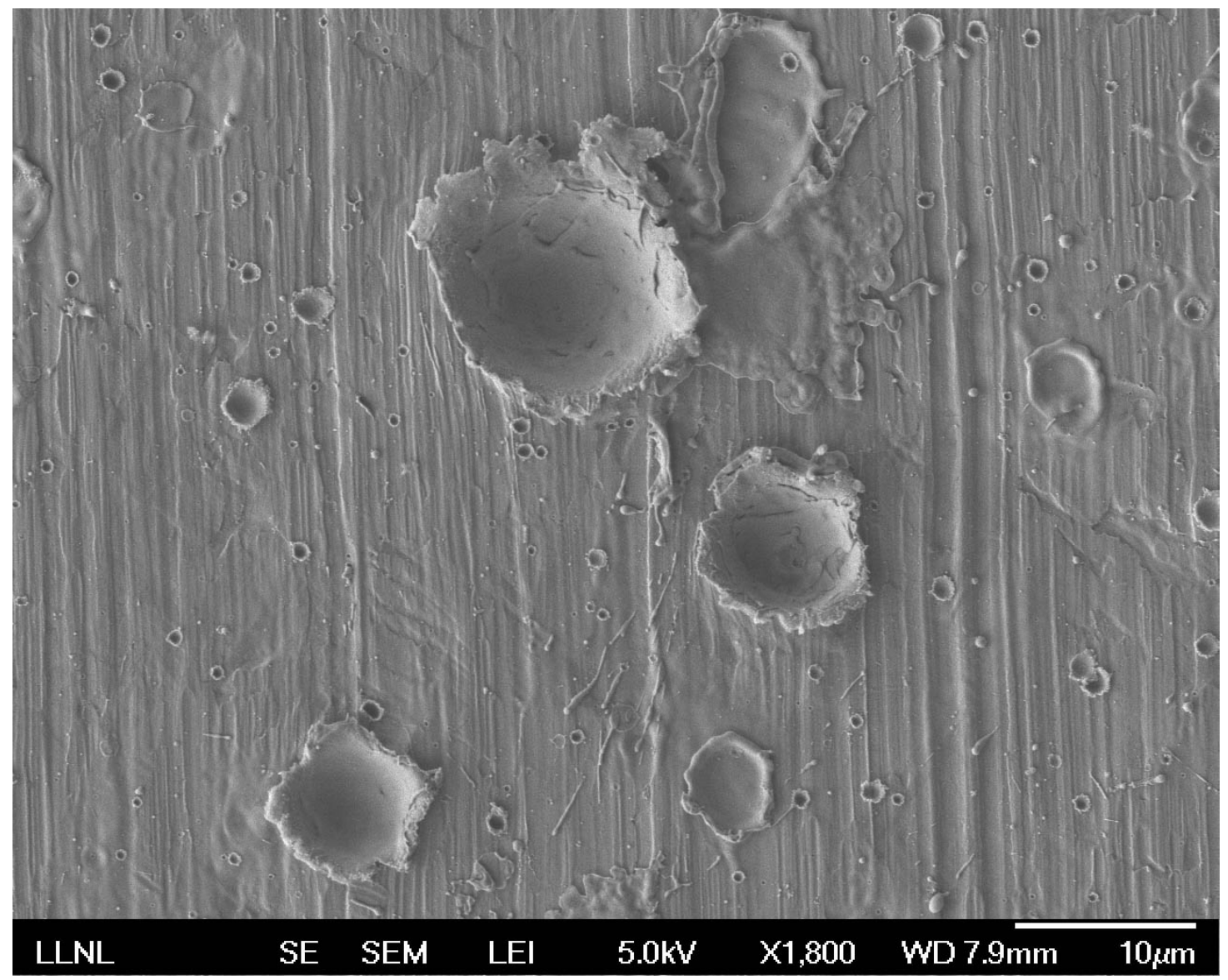

Fig. 8.- Another section of the same $\mathrm{Cu}$ grain catcher foil with examples of craters containing $\mathrm{Al}$ and $\mathrm{O}$, which can only have come from dust grain impacts. [The image is a lower secondary electron image (LEI) produced by a scanning electron microscope (SEM) at $1800 \times$ magnification.] 


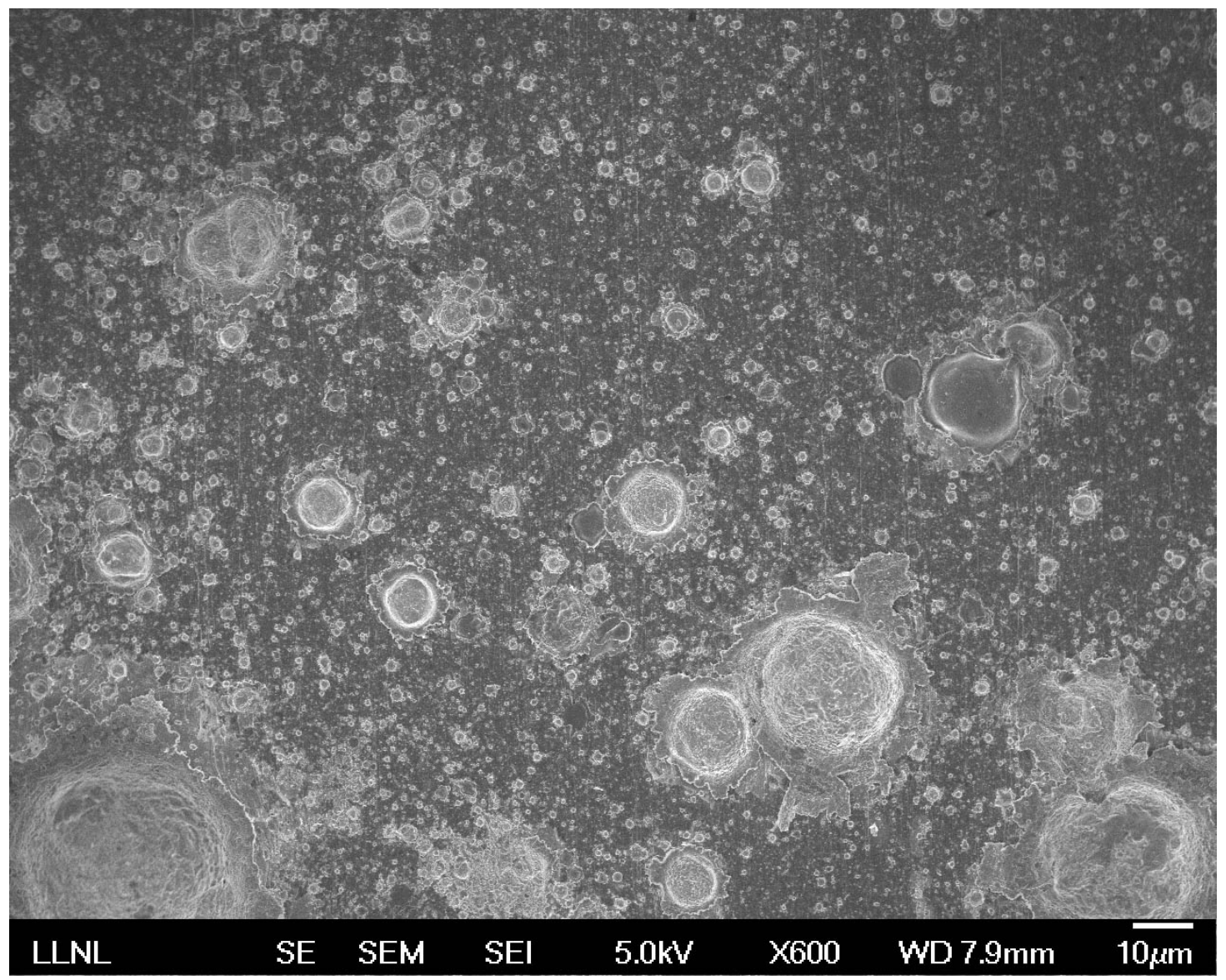

Fig. 9. - Some of the craters in this area of the foil contain Fe, suggesting they were caused by impacts of droplets coming off the target hole walls. [The image is a secondary electron image (SEI) produced by a scanning electron microscope (SEM) at $600 \times$ magnification.] 
to and remained in the diamond phase on this short time scale (21).

This work was performed under the auspices of the U.S. Department of Energy by Lawrence Livermore National Laboratory under Contract DE-AC52-07NA27344.

Facilities: Jupiter Laser Facility

\section{REFERENCES}

J. S. Mathis, W. Rumpl, and K. H. Nordsieck, Size distribution of interstellar grains, Astrophys. J. 217, 425 (1977).

B. T. Draine and E. E. Salpeter, Destruction mechanims for interstellar dust, Astrophys. J. 231, 438 (1979).

C. Gregory Seab and J. Michael Shull, Shock processing of interstellar grains, Astrophys. J. 275, 652 (1983).

A. G. G. M. Tielens, C. G. Seab, D. J. Hollenbach, and C. F. McKee, Shock processing of interstellar dust: diamonds in the sky, Astrophys. J. 319, L109 (1987).

C. F. McKee, D. J. Hollenback, C. G. Seab, and A. G. G. M. Tielens, The structure of time-dependent interstellar shocks and grain destruction in the interstellar medium, Astrophys. J. 318, 674 (1987).

A. P. Jones, A. G. G. M. Tielens, D. J. Hollenback, and C. F. McKee, Grain destruction in shocks in the interstellar medium, Astrophys. J. 433, 797 (1994).

D. A. Neufeld, S. Lepp, and G. J. Melnick, Thermal balance in dense molecular clouds: radiative cooling rates and emission-line luminosities, Astrophys. J. Suppl. S. 100, 132 (1995).

A. P. Jones, A. G. G. M. Tielens, and D. J. Hollenback, Grain shattering in shocks: the interstellar grain size distribution, Astrophys. J. 469, 740 (1996).

R. S. Lewis, M. Tang, J. F. Wacker, E. Anders, and E. Steel, Interstellar diamonds in meteorites, Nature 326, 160 (1987).

N. McBride, S. F. Green, and J. A. M. McDonnell, Meteoroids and small sized debris in low earth orbit and at 1 AU: results of recent modeling, Adv. Space. Res. 23, 73 (1999). 
G. A. Graham, A. T. Kearsley, M. M. Grady, I. P. Wright, A. D. Griffiths, and J. A. M. McDonnel, Hypervelocity impacts in low earth orbit: cosmic dust versus space debris, Adv. Space Res. 23, 95 (1999).

M. J. Burchell, M. J. Cole, J. A. M. McDonnell, and J. C. Zarnecki, Meas. Sci. Technol. 10, 41 (1999).

F. Horz, M. E. Zolensky, R. P. Bernhard, T. H. See, and J. L. Warren, Impact features and projectile residues in aerogel exposed on Mir, Icarus 147, 559 (2000).

G. A. Graham, N. McBride, A. T. Kearsley, G. Drolshagen, S. F. Green, J. A. M. McDonnell, M. M. Grady, and I. P. Wright, The chemistry of micrometeoroid and space debris remnants captured on Hubble Space Telescope solar cells, Int. J. Impact Eng. 26, 263 (2001).

G. A. Graham, A. T. Kearsley, G. Drolshagen, N. McBride, S. F. Green, and I. P. Wright, Microparticle impacts upon HST solar cells, Adv. Space Res. 28, 1341 (2001).

G. A. Graham, A. T. Kearsley, I. P. Wright, M. J. Burchell, and E. A. Taylor, Observations on hypervelocity impact damage sustained by multi-layered insulation foils exposed in low earth orbit and simulated in the laboratory, Int. J. Impact Eng. 29, 307 (2003).

G. A. Graham, P. G. Grant, R. J. Chater, A. J. Westphal, A. T. Kearsley, C. Snead, G. Dominguez, A. L. Butterworth, D. S. McPhail, G. Bench, and J. P. Bradley, Investigation of ion beam techniques for the analysis and exposure of particles encapsulated by silica aerogel: Applicability for Stardust, Meteorit. Planet. Sci. 39, 1461 (2004).

J. T. Larsen and S. M. Lane, Hyades - A plasma hydrodynamics code for dense-plasma studies, J. Quant. Spectrosc. Radiat. Transfer 51, 179 (1994).

http://jlf.llnl.gov

Comet chasers get mineral shock, Nature 440, 260 (2006).

G. A. Graham, N. Teslich, Z. R. Dai, J. P. Bradley, A. T. Kearsley, F. Horz, Focused ion beam recovery of hypervelocity impact residue in experimental craters on metallic foils, Meteorit. Planet. Sci. 41, 159 (2006). 